\title{
FIFA World Cup 2006 in Germany: enhanced surveillance improved timeliness and detection
}

\author{
C. J. WILLIAMS ${ }^{1,2 *}$, K. SCHENKEL ${ }^{1}$, T. ECKMANNS ${ }^{1}$, D. ALTMANN ${ }^{1}$ \\ AND G. KRAUSE ${ }^{1}$ \\ ${ }^{1}$ Department for Infectious Disease Epidemiology, Robert Koch Institute, Berlin, Germany \\ ${ }^{2}$ European Programme for Intervention Epidemiology Training (EPIET), European Centre for Disease \\ Prevention and Control (ECDC), Stockholm, Sweden
}

(Accepted 28 June 2008; first published online 31 July 2008)

\section{SUMMARY}

Enhanced surveillance for infectious disease events, with accelerated routine reporting and daily supplementary reports, was undertaken during the 2006 FIFA World Cup in Germany. We evaluated the surveillance outputs, reporting intervals and detection of World Cup-relevant events for the enhanced system. Outbreak numbers for measles, Norovirus and Campylobacter were significantly higher than in previous years, but all increases were explained by prior trends. The median interval (disease onset to receipt at national centre) fell from 17 days in 2005 to 12 days in 2006. Detection of World Cup-relevant events was $44 \%(8 / 18)$ in the routine system and $77 \%(14 / 18)$ in supplementary reports. We did not identify any significant effect on infectious disease epidemiology relating to the FIFA 2006 World Cup. Daily reporting improved timeliness, and supplementary reporting improved relevant event detection. Enhancing existing systems, without the addition of syndromic surveillance, can be an effective approach to mass-event surveillance.

Key words: Event surveillance, mass gatherings, 2006 FIFA World Cup, Germany, surveillance.

\section{INTRODUCTION}

\section{World Cup 2006}

The 2006 FIFA (Fédération International de Football Association) World Cup was held in Germany between 9 June and 9 July 2006. Sixty-four matches were held in 12 cities from nine German states, with around 3 million stadium tickets sold [1]. Based on these ticket sales around 1 million foreign visitors were anticipated. Around 21 million people were estimated to have attended the official fan festivals, where games

\footnotetext{
* Author for correspondence: Dr C. J. Williams, 120 Bromedale Avenue, Mulbarton, Norfolk NR14 8GZ, UK.

(Email: kitwilliams@doctors.org.uk)
}

were shown on large screens, with 9 million in Berlin alone [2]. Additional foreign visitors not carrying tickets, and visitors from other parts of Germany, were also expected, and visitors and residents gathered to watch matches in bars and cafes.

\section{Event surveillance}

At any event where people gather, there is the potential for both non-infectious health hazards including injury [3], exacerbations of pre-existing disease [4], and heat-related illness [5]; and infectious disease events.

Infectious disease transmission may be promoted by an increase in population density, importation of unusual pathogens, strains on infrastructure, and 
changes in services (such as food stalls) or behaviour (increased demand for sex workers). Large events are also potential targets for bioterrorism [6].

There have been some reports of infectious disease outbreaks at mass events. Syndromic surveillance at an outdoor camping event in the United States identified outbreaks of gastroenteritis [5]. A cluster of meningococcal meningitis cases in the United Kingdom were linked to attendance at a rugby match [7], and meningitis outbreaks have been linked to the annual pilgrimage to Mecca (the Hajj) [8].

In view of this background, the Robert Koch Institute (RKI) in Berlin (national communicable disease institute) decided to institute enhanced surveillance but not additional syndromic surveillance. The main reasons for the provision of enhanced surveillance were the perceived increased risk of infectious disease events and the need to detect and respond to such events quickly due to their short-lived nature.

We analysed the results of the enhanced surveillance in order to determine whether that the FIFA 2006 World Cup had had an effect on infectious disease epidemiology in Germany, and evaluated the enhanced system itself in order to assess its added value in such mass events.

\section{METHODS}

\section{Enhanced surveillance activities}

Germany already has an electronic reporting system for infectious diseases, SurvNet, whose design and characteristics have been recently described [9]. Rather than creating a new system for the event, it was decided to strengthen and augment the existing surveillance structures.

The enhanced surveillance measures were undertaken in the local and state health departments responsible for the 12 cities hosting tournament matches (World Cup cities: Berlin, Cologne, Dortmund, Frankfurt, Gelsenkirchen, Hamburg, Hannover, Kaiserslautern, Leipzig, Munich, Nuremberg, Stuttgart), during the period 7 June to 11 July 2006. The 2 days of surveillance immediately before the match was designed to provide a short run-in period for the system, and the 2 days following the final match to allow capture of the commonest, short-incubation period gastrointestinal infections.

There were four main enhancements to infectious disease surveillance during the FIFA 2006 World
Cup: accelerated transmission of notifiable disease case-data (from weekly to daily); marking cases of relevance to the FIFA 2006 World Cup in routine notifications (SurvNet); daily supplementary reports from all cities hosting matches; and the National Enhanced Surveillance Operations Centre (NESOC), staffed Monday to Saturday, in the national epidemiology centre (RKI) which collected, analysed and reported on daily surveillance inputs. Daily supplementary reports were structured using four headings: disease outbreaks; unusual individual cases of notifiable diseases; other unusual individual cases; and any other unusual events. These were reported briefly in Word format on a daily basis and were intended as a supplementary instrument to promptly capture events that would not fulfil the case definition of the routine reporting system.

Communication with local and state health departments was facilitated by teleconferences, email and telephone communications, and pre-World Cup training sessions. A commercial text search for 14 infection-related keywords including 'bacteria', 'epidemic' and 'infection', of 25 German newspapers was used to screen for relevant press reports. Further details of this enhanced surveillance were published in $2006[10]$.

\section{Data from routine reporting system (SurvNet)}

All SurvNet data extracted (but not the routine surveillance itself) were restricted to the 47 pathogens or diseases notifiable through local health departments, and to the World Cup cities listed above, apart from where otherwise specified. Pathogens or diseases directly notifiable to the RKI (bypassing local health departments) were excluded from the evaluation (but not surveillance activities) as we were concerned with the interactions between the local health departments in the World Cup cities and the RKI surveillance activities.

SurvNet is a working surveillance system and data is constantly updated and revised; therefore data extractions can specify the time point at which they are valid. All SurvNet data used was valid as of 1 August 2006 (3 weeks after the tournament period) except for 2007 outbreak data, which was valid at 1 August 2007. All time comparisons used SurvNet data from weeks 23 to 29 inclusive, a 7-week period from 5 June (4 days before the first match) to 23 July 2006 ( 2 weeks after the last match), in order to capture earlier or later notified cases. 


\section{Numbers of notified cases and outbreaks reported to SurvNet}

We extracted data on SurvNet case notifications from 2001 to 2006, and also on outbreaks notified to SurvNet from 2001 to 2007. Data obtained included fulfilment of RKI reference definitions, year of reporting, and organism. The populations of the 12 World Cup cities were obtained from the German Statistical Office [11]. For pathogens or diseases with three or more outbreaks in 2006 we calculated Poisson confidence intervals for the years 2001 to 2005 using Intercooled Stata, version 9 (StataCorp, College Station TX, USA), and compared these with the figures for 2006.

\section{Comparison of reported disease burden with expected burden}

In order to estimate the number of additional cases of selected infections that might result from the increase in tourism, we calculated the background incidence of Campylobacter, Salmonella and Norovirus per person day, for weeks 23-29 in the 12 cities hosting matches, based on 2001-2005 figures (2005 only for Norovirus due to a rising secular trend).

These pathogen-specific incidences were multiplied by the estimated additional overseas overnight visitors to Germany during the World Cup, obtained from a report published by the German tourism office [12], cross-referenced with a report from the German Statistical Office [13].

\section{Detection of WM-relevant events}

We extracted case-data for all notifications to SurvNet marked as 'WM-2006' in the free text of the form, including those from local health departments outside the World Cup cities listed above. All events from the daily status reports were entered into a spreadsheet, classified by columns including organism, number of cases, health department and supplementary description. It should be noted that reporting offices were not given a strict definition of cases which should be reported in the supplementary system.

An event was defined as the first report of one or more linked cases in the routine (SurvNet) or supplementary reporting systems. A FIFA 2006 World Cup-marked event was defined as one which contained the marker 'WM-2006' in the SurvNet system, or (in the supplementary reports) as any event which mentioned the World Cup, a FIFA 2006 World Cup team or any other event linked to the FIFA 2006 World Cup in the text. A foreign visitor-related event or case was defined as any FIFA 2006 World Cupmarked case in SurvNet in a foreign national, or as any event in the supplementary daily report where foreign nationality was recorded.

A FIFA 2006 World Cup-relevant event (or case) was defined as any event (or case) which was either FIFA 2006 World Cup-marked or foreign visitorrelated. Using inclusion in either source as the 'gold standard', we estimated the detection of these FIFA 2006 World Cup-relevant events by each system (supplementary reports and SurvNet).

\section{Timeliness and data quality}

We extracted the dates of disease onset, diagnosis, notification, receipt at local health department, and receipt at RKI on cases reported to SurvNet subject to the above restrictions. Three time intervals were calculated using Stata: disease onset to receipt at RKI; diagnosis to notification; and entry in local health department to receipt at RKI. We excluded observations with intervals exceeding 365 days (onset to RKI receipt) or 100 days (latter two intervals).

We compared the mean, median and 10th and 90th percentiles for each interval in 2006 and 2005, using the Mann-Whitney test for the statistical significance of differences.

The percentage of notified cases fulfilling the most specific of the RKI reference definitions was used as an estimator of data quality, with data from 2005 as the comparator.

We looked for and documented examples of reporting delay by comparing daily supplementary reports, SurvNet data, press reports collated during the FIFA 2006 World Cup period, and feedback from the RKI surveillance team.

\section{Comparison of reporting to supplementary and routine (SurvNet) systems}

The proportion of cases notified to SurvNet from which were included in daily supplementary reports was calculated for each notifiable organism.

\section{Additional resources used at local and national level}

A web-based questionnaire survey of the 12 local health departments covering the 12 FIFA 2006 World Cup cities was conducted just after the 1-week test 
period for the enhanced surveillance. Health departments were asked how long they had spent per day on researching cases and on data transmission and supplementary reports. They were also asked about additional staff recruited and additional resources provided.

We used the mid-point of each time category (e.g. $90 \mathrm{~min}$ for the $1-2 \mathrm{~h}$ category) to calculate summary statistics. RKI staff resources were calculated using the daily surveillance team roster.

\section{RESULTS}

\section{Numbers of notified cases and outbreaks reported to SurvNet}

There were 4112 (3793 fulfilling reference definitions) cases notified to the routine surveillance system SurvNet from the 12 match-hosting cities between weeks 23 and 29, fewer than the 5083 (4889 fulfilling reference definitions) from the same period in 2005.

The commonest outbreak-causing organisms $(>2$ outbreaks in 2006) in weeks 23-29 of 2005 and 2006 were Salmonella, Campylobacter, Norovirus, Rotavirus, Giardia and measles. During the FIFA 2006 World Cup period, Campylobacter, Norovirus and measles outbreak numbers exceeded the upper Poisson confidence interval for the same cities and period during 2001-2005.

Figure 1 shows the number of outbreaks caused by these three organisms in the same period in years 2001-2007. The increase in Norovirus and Campylobacter outbreaks is consistent with an increasing secular trend, the latter decreasing after 2005. Only for measles is the increased number of outbreaks (four) not part of a broader trend.

\section{Expected cases in foreign visitors}

Non-German nationals accounted for an estimated two million additional overnight stays during the FIFA 2006 World Cup period.

This population time would be expected to give rise to an additional six Campylobacter, five Salmonella and two Norovirus cases (Table 1).

Actual reports of Salmonella, Campylobacter and Norovirus in foreign nationals amounted to two Campylobacter cases (one from SurvNet, one additionally from supplementary reports), six Norovirus cases (SurvNet) and three Salmonella cases (SurvNet).

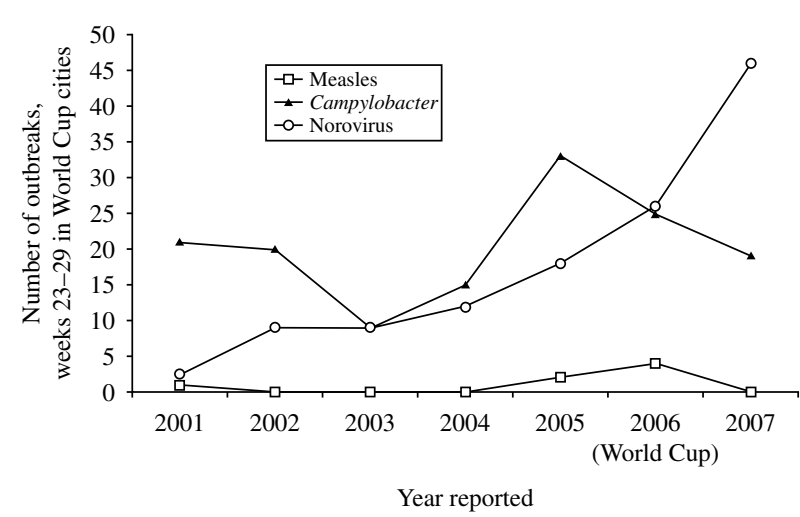

Fig. 1. Number of reported outbreaks due to selected diseases, 2001-2007, weeks 23-29 in World Cup cities.

\section{Detection of FIFA 2006 World Cup-relevant events in supplementary reports and SurvNet}

There were 155 events in the supplementary reports. The commonest causative organisms (at least four reported events) were measles, scarlet fever, chickenpox, hand foot and mouth disease, erythema infectiosum, legionellosis, Norovirus, gastroenteritis, pertussis and Salmonella.

Seventy-one FIFA 2006 World Cup-marked cases from eight events were reported to SurvNet, of which 63 were Norovirus cases related to the Munich broadcasting company outbreak. The remaining eight comprised four Salmonella and four Campylobacter cases (two of the latter being part of the same event).

Including the Munich Norovirus outbreak, 14 FIFA 2006 World Cup-marked events (77 cases) were notified via either the daily supplementary reports or the SurvNet system. Four were reported to SurvNet but not in supplementary reports: all were from health departments not participating in the enhanced surveillance. Six were reported in the supplementary report but not in SurvNet, of which four involved non-German nationals, and two were non-notifiable, non-infectious hazards (pollen allergy and a hailstorm).

There were 11 events (30 cases) reported in nonGerman nationals: nine single cases, and two outbreaks involving foreign visitors (total 21 cases). These included cases of chickenpox, malaria, Legionella, gastroenteritis and one case of mumps. No cases of measles in non-German nationals were reported to either system.

A total of 18 events from SurvNet and the supplementary reports were categorized as FIFA 2006 
Table 1. Estimated additional cases in non-German nationals during the World Cup period due to additional overnight stays, Campylobacter, Salmonella and Norovirus

\begin{tabular}{llll}
\hline \hline & Campylobacter & Salmonella & $\begin{array}{l}\text { Norovirus (based } \\
\text { on 2005 figures only) }\end{array}$ \\
\hline $\begin{array}{c}\text { Mean cases per year in weeks } \\
\text { 23-29 (2001-2005) }\end{array}$ & 1718 & 1379 & 557 \\
$\begin{array}{c}\text { Mean cases per day in weeks } \\
\text { 23-29 (2001-2005) }\end{array}$ & $0 \cdot 30$ & $0 \cdot 24$ & $0 \cdot 10$ \\
$\begin{array}{c}\text { Extra person-days in 2006 } \\
\text { Estimated extra cases, 2006 }\end{array}$ & 2000000 & 2000000 & 2000000 \\
$\begin{array}{c}\text { Observed cases (SurvNet and } \\
\text { supplementary reports }\end{array}$ & 2 & 5 & 2 \\
\hline \hline
\end{tabular}

Table 2. Time periods between notification steps, World Cup cities, weeks 23-29, 2006 compared to 2005 (SurvNet data, data as of 1 August 2006)

\begin{tabular}{llll}
\hline \hline Time period (days) & Statistics & $\begin{array}{l}\text { 2005 weeks 23-29 } \\
\text { (control period) }\end{array}$ & $\begin{array}{l}\text { 2006 weeks 23-29 } \\
\text { (World Cup period) }\end{array}$ \\
\hline Diagnosis to notification & Mean (number of observations) & $2 \cdot 82(3365)$ & $2 \cdot 65(2536)$ \\
& Median (10th-90th percentile) & $2(0-6)$ & $1(0-6)$ \\
$\begin{array}{l}\text { Entry in local health } \\
\text { department to }\end{array}$ & Mean (number of observations) & $5 \cdot 9(4788)$ & $3 \cdot 08(3704)$ \\
$\begin{array}{r}\text { receipt in RKI } \\
\begin{array}{l}\text { Illness onset to receipt } \\
\text { in RKI }\end{array}\end{array}$ & Median (10th-90th percentile) & $3(1-9)$ & $1(0-6)$ \\
\hline \hline
\end{tabular}

RKI, Robert Koch Institute.

World Cup-relevant (i.e. either FIFA 2006 World Cup-marked or foreign visitor-related). The detection of FIFA 2006 World Cup-relevant events was $44 \%$ $(8 / 18)$ in SurvNet and $78 \%(14 / 18)$ in the supplementary reports.

\section{Timeliness and data quality}

We used 5901/6213 cases for diagnosis to notification calculations; 8492/8492 for entry in local health department to receipt at RKI; and 5512/5524 for disease onset to receipt at RKI. Table 2 shows the summary statistics for the three time intervals.

All time intervals were lower in 2006 compared to 2005. Overall time from disease onset to receipt at RKI fell from a median of 17 days to a median of 12 days (2006). The reductions were significant for all three time periods (Mann-Whitney test, $P<0 \cdot 01$ ).

The percentage of cases fitting the reference definition was significantly lower during the surveillance period $(3127 / 4112,76 \cdot 0 \%)$ than in the same period in $2005(4106 / 5083,80 \cdot 8 \%)(P<0 \cdot 001)$.

\section{Early detection through press scanning}

There were several incidents where press reports identified an issue before it was notified to RKI through the enhanced surveillance system.

These included a case of meningococcal disease in Bavaria (not FIFA 2006 World Cup-related); gastrointestinal illness in the Croatian national football team (event 2 June 2006, press report 3 June 2006 [14], in RKI report 9 June 2006); and cases of chickenpox in a Togo national team player, and in an Indonesian journalist (press report 30 May 2006, RKI report 8 June 2006). The latter two press reports were identified through ad-hoc reporting rather than the daily press screening. Only three of the 26 reports identified in the German local press by the press screening had any relation to the 2006 FIFA World Cup. 
Table 3. Cases reported in local health department daily supplementary reports (7 June 2006 to 11 July 2006) compared with SurvNet notifications (weeks 23-29, 2006, cases fulfilling reference definition, data as of 1 August 2006) in World Cup cities

\begin{tabular}{|c|c|c|c|c|}
\hline Organism & $\begin{array}{l}\text { Total reports } \\
\text { (events) (A) }\end{array}$ & $\begin{array}{l}\text { Total affected } \\
\text { (cases) (B) }\end{array}$ & $\begin{array}{l}\text { Number notified } \\
\text { to Survnet }(\mathrm{C})\end{array}$ & $\begin{array}{l}\% \text { SurvNet cases } \\
\text { in supplementary } \\
\text { reports }(\mathrm{B} / \mathrm{C})\end{array}$ \\
\hline Measles* & 32 & 134 & 48 & $279 \cdot 2 \%$ \\
\hline Hepatitis A & 7 & 8 & 46 & $17 \cdot 4 \%$ \\
\hline Legionella & 7 & 7 & 16 & $43 \cdot 8 \%$ \\
\hline Norovirus & 6 & 72 & 429 & $16 \cdot 8 \%$ \\
\hline Salmonella & 4 & 5 & 877 & $0.6 \%$ \\
\hline $\begin{array}{l}\text { Tick-borne } \\
\text { encephalitis* }\end{array}$ & 3 & 7 & 4 & $175.0 \%$ \\
\hline $\begin{array}{l}\text { Haemolytic uraemic } \\
\text { syndrome* }\end{array}$ & 3 & 3 & 2 & $150 \cdot 0 \%$ \\
\hline Shigellosis & 3 & 3 & 17 & $17 \cdot 6 \%$ \\
\hline Campylobacter & 2 & 2 & 1207 & $0 \cdot 2 \%$ \\
\hline Meningitis & 2 & 2 & 5 & $40 \cdot 0 \%$ \\
\hline Hantavirus & 1 & 1 & 2 & $50 \cdot 0 \%$ \\
\hline Listeriosis & 1 & 1 & 8 & $12 \cdot 5 \%$ \\
\hline Rotavirus & 1 & 5 & 255 & $2.0 \%$ \\
\hline
\end{tabular}

* Organisms or diseases in bold are those where the number of cases in the supplementary reports exceeds those reported to SurvNet.

\section{Comparison of reporting to supplementary and routine (SurvNet) systems}

Table 3 compares the number of reports and cases for a selection of organisms featuring in both SurvNet notifications and the local health department daily supplementary reports.

For three organisms (measles, tick-borne encephalitis and haemolytic-uraemic syndrome: bold in Table 3), more cases were reported in daily supplementary reports than in SurvNet. For other organisms, the proportion of routine cases reported in supplementary reports varied from $0.2 \%$ to $0.6 \%$ (Campylobacter and Salmonella respectively) to $50 \%$ (Hantavirus).

\section{Resources used}

\section{RKI}

The RKI team consisted of two staff, drawn on a rotating basis from the department. The daily shift was from 09:00 to 15:30 hours $(6 \cdot 5 \mathrm{~h})$ apart from Saturday (09:00-13:00 hours), 6 days per week, and ran during the test week prior to the event and then for 5 weeks during the FIFA 2006 World Cup (7 June 2006 to 11 July 2006). A room was assigned for the duration of the surveillance, and was supplied with telephones, a fax machine, laptop computers and a television.

\section{Local health departments}

The 13/13 local health departments who answered the pre-FIFA 2006 World Cup survey required a median of 30-60 min per day (range $<30 \mathrm{~min}$ to $2-4 \mathrm{~h}$ ) for investigating FIFA 2006 World Cup-relevant cases, and a further 30-60 min median for data transmission and daily supplementary report compilation (range $<30$ min to $1-2 \mathrm{~h}$ ) during the test week.

One local health department required additional staff for the enhanced surveillance. Three out of 13 local health departments required additional nonpersonnel resources for the enhanced surveillance preparation (one $<€ 500$, one $€ 500-1000$, one $>€ 1000-5000$ ).

No department received additional funding for the enhanced surveillance. Suggested uses for additional resources (had they been provided) included extra staff and training, preparation of information materials, improvement of on-call functions, and payment for overtime and extra duties.

\section{DISCUSSION}

During the 2006 FIFA World Cup there was no increase in reported infectious episodes, and the expected number of additional cases was low. The increases in the numbers of outbreaks due to 
Norovirus, Campylobacter and measles were either part of a secular trend (Norovirus and Campylobacter) or due to a large outbreak which predated the 2006 FIFA 2006 World Cup [15] (measles). Enhanced surveillance improved timeliness and detection of relevant events, without requiring significant extra resources.

This absence of evidence of any increase in overall infectious disease events is consistent with the findings from other event surveillance reports. At the EURO 2004 football tournament in Portugal, no visitors were found to be affected by infectious disease outbreaks, and the number of outbreaks in the local population did not exceed expected values based on data from the preceding 3 years [16]. At the Sydney Olympics in 2000, the number of infectious disease notifications was in line with the numbers from the preceding and following years [17]. A similar picture was reported from surveillance during the millennium year in Rome [18].

The expected additional numbers of Norovirus, Campylobacter and Salmonella infections in foreign visitors were low compared to total notifications of these organisms during the tournament period (13 expected; 2513 notified). Even if the risk of infection in foreign visitors was higher than the background rate, foreign cases would still be greatly outnumbered by local cases.

The only significant outbreak involving foreign visitors was the Munich Norovirus outbreak which involved at least five foreign nationals. The 30 cases (11 events) in non-German nationals reported through enhanced surveillance was a small proportion of the total notifications $(0.7 \%$ of 4112$)$ in the same period. A similarly low proportion of notifications in non-residents $(12 / 1752,0.7 \%)$ was found at the Sydney Olympics [17]. During the millennium year in Rome, the numbers of Legionella cases and foodborne outbreaks in foreign visitors were higher than expected levels, but the number of visitors (26 million) and the period of surveillance (1 year) were much greater than for the World Cup and Sydney Olympics.

Supplementary reporting increased detection of FIFA 2006 World Cup-relevant events, which might otherwise have gone unremarked in the routine system. Apart from the Munich Norovirus outbreak, very few FIFA 2006 World Cup-marked cases were reported to SurvNet. However, many cases and events in the supplementary daily reports, such as cases of viral diseases in day-care centres for young children, were not relevant to the event. Thus the increased sensitivity came at the cost of a reduced positive predictive value.

The threshold for reporting events in the daily supplementary reports varied widely and was influenced by topicality. For example, following a PanAmerican Health Organisation (PAHO) warning about the measles outbreak in Germany, the RKI raised awareness among participating health departments of the importance of notifying measles cases. This accounts for the high number of measles cases in the supplementary reports.

The move to daily transmission of routine notifications reduced the total reporting interval by 5 days, which is significant during an event lasting only 30 days. Faster transmission may account for the small reduction in data quality, with cases being transmitted before information gathering is complete. The few instances where cases were first identified through mass media justified the daily media screening.

There were several limitations in the evaluation of the enhanced surveillance. There is no single definition of a 'FIFA 2006 World Cup-relevant' case, and even if there were, it would be impossible to determine the true number of such events to assess the sensitivity of any enhanced surveillance. As we had no strict definition of event relevance, local departments had the opportunity to report anything they felt to be significant, with the aim of improving sensitivity for important events.

The low number of cases reported in non-German nationals may be due to visitors having poorer access to health care, or not becoming symptomatic and/or seeking medical help until their return home. Our application of background risk when calculating estimated cases of Norovirus, Campylobacter and Salmonella in foreign visitors may have been incorrect, if risk behaviour were different among visitors compared to the host population.

Despite the existence of potential drivers of infectious disease transmission during mass events, our results add to the body of evidence suggesting that mass events do not increase the burden of infectious disease in either the host or visitor populations. This may be because visitors to such events are relatively affluent, young and healthy and are therefore less likely to fall ill.

However, enhanced surveillance at such events is likely to continue as a reassurance for governing authorities and the public especially in light of the possibility of bioterrorism attacks during such highprofile events. 
Syndromic surveillance has been employed at many recent international mass gatherings, including the FIFA World Cup in 1998 (France) [19]; the Olympic games in 2004 (Athens) [20] and 2000 (Sydney); the Winter Olympics in Turin (2006) [21] and Salt Lake City (2002) [22]; and G8 summits in Japan (2000) [23] and the United Kingdom (2005) [24]. Of the signals detected by syndromic surveillance at these events, only two were confirmed as outbreaks or clusters [23, 24]; no other significant infectious disease events were identified. In two cases outbreaks of Legionella [19] and gastrointestinal disease [23] not detected using syndromic surveillance were identified through the usual notification system. Thus syndromic surveillance may not always be the best response to infectious disease surveillance at international events.

Syndromic surveillance involves a system additional to the routine notification system which captures data on sets of presenting symptoms or syndromes from other health-care sources such as primary care or emergency departments, and analyses this separate dataset to detect signals which may indicate infectious disease cases or outbreaks. In the absence of a strong and timely routine notification system, syndromic surveillance can be extremely useful for mass events.

Syndromic surveillance was not implemented at the FIFA 2006 World Cup due to the resource demands of such systems, the strengths of the existing routine notification system, and the possibility of using the efforts for the event to improve the routine system. An additional system would perhaps have diverted resources from the existing one, and required additional resources to reconcile differing data signals from the two systems. The existing routine system worked well, and already allowed the reporting of outbreaks of particular syndromes before pathogen isolation. Moreover, enhanced surveillance offered a chance to trial accelerated routine reporting, which could then be extended to improve the whole routine system for the future.

The surveillance evaluation suggests that satisfactory event surveillance can be achieved through minor temporary adaptations of an existing routine infectious disease surveillance system. Our approach enables good comparability with data from other areas and time periods, but also means that the extra efforts required for a mass gathering can be harnessed for longer-term improvements in routine surveillance.

Potential drivers for increased infectious disease transmission occur commonly, due to commuting, seasonal travel and smaller-scale gatherings such as concerts and conferences. A timely, sensitive and reliable surveillance system is therefore invaluable in the detection of associated problems at all times, not just during international sporting events.

\section{ACKNOWLEDGEMENTS}

Many thanks to the participating community health authorities of Berlin, Cologne, Dortmund, Frankfurt, Gelsenkirchen, Hamburg, Hannover, Kaiserslautern, Leipzig, Munich, Nuremberg, Stuttgart; the state health departments of Bavaria, Berlin, Hamburg, Hesse, North Rhine Westphalia, Lower Saxony, Rhineland-Palatine and Saxony; the staff of the Robert Koch Institute who participated in the enhanced surveillance; Robert Koch Institute Press Department; Justin Josephson, Justus Benzler, Gabi Poggensee, Katharina Alpers and Matthias AnderHeiden (Robert Koch Institute); Marta Valenciano and Arnold Bosman (EPIET).

\section{DECLARATION OF INTEREST}

None.

\section{REFERENCES}

1. Ahlert G. Auswirkungen des zusätzlichen IncomingTourismus während der FIFA WM 2006 (GWSInstitute of Economic Structures Research website 2006) (http://www.gws-os.de/Downloads/gws-paper061.pdf). Accessed 16 July 2008.

2. Deutsche Zentrale für Tourismus. Effekte der FIFA WM 2006: Ein Volltreffer für den DeutschlandTourismus (German tourism centre website 2007) (http://www.deutschland-extranet.de/pdf/pm_wm_ bilanz 070514.pdf). Accessed 16 July 2008.

3. Zeitz $\overline{\mathbf{K}}$. Injury occurrences at a mass gathering event. Journal of Emergency Primary Health Care 2005; 3: $1-2$.

4. Ahmed QA, Arabi YM, Memish ZA. Health risks at the Hajj. Lancet 2006; 367: 1008-1015.

5. CDC. Surveillance for early detection of disease outbreaks at an outdoor mass gathering - Virginia, 2005. Morbidity and Mortality Weekly Report 2006; 55: $71-74$.

6. Inglesby TV. Anthrax : a possible case history. Emerging Infectious Diseases 1999; 5: 556-560.

7. Orr $\mathbf{H}$, et al. Cluster of meningococcal disease in rugby match spectators. Communicable Disease and Public Health 2001; 4: 316-318.

8. Aguilera JF, et al. Outbreak of serogroup W135 meningococcal disease after the Hajj pilgrimage, Europe, 2000. Emerging Infectious Diseases 2002; 8: 761-767. 
9. Krause G, et al. SurvNet electronic surveillance system for infectious disease outbreaks, Germany. Emerging Infectious Diseases 2007; 13: 10.

10. Schenkel K, et al. Enhanced surveillance of infectious diseases: the 2006 FIFA World Cup experience, Germany. Eurosurveillance Monthly 2006; 11: 234-238.

11. Federal Statistical Office Germany. Genesis-online statistical information system. (German federal statistical office website 2007) (https://www-genesis. destatis.de/genesis/online/logon). Accessed November 2007.

12. Deutsche Zentrale für Tourismus, Deu. Effekte der FIFA WM 2006: DZT wertet Fußball-WM als Volltreffer (German tourism centre website 2006) (http:// www.deutschland-extranet.de). Accessed November 2007.

13. Graffy C. $2 \%$ mehr Übernachtungen im Juli 2006. Statistisches Bundesamt Deutschland (Press release no. 369 2006) (http://www.destatis.de/jetspeed/portal/cms/ Sites/destatis/Internet/DE/Presse/pm/2006/09/PD06_ 369_454.psml). Accessed 16 July 2008.

14. RTÉ.ie. Smolarek strike boosts Polish morale (RTÉ.ie news website 2006) (http://rte.ie/pda/sport/2006/0603/ 1094620.html). Accessed 16 July 2008.

15. van Treeck U. Measles outbreak in Germany: over 1000 cases now reported in Nordrhein Westfalen. Eurosurveillance Weekly 2006; 11(5): E060511.

16. Goncalves $\mathbf{G}$, et al. Infectious diseases surveillance activities in the north of Portugal, during the EURO 2004 football tournament. Eurosurveillance Monthly 2005; 10: $86-89$.
17. Jorm LR, et al. Watching the Games: public health surveillance for the Sydney 2000 Olympic Games. Journal of Epidemiology and Community Health 2003; 57: 102-108.

18. Giorgi RP, et al. Infectious diseases in Rome during the Millennium year. Eurosurveillance Monthly 2003; 8: 181-185.

19. Hanslik T, et al. Sentinel monitoring of general community health during the 1998 World Football Cup. Revue d'épidémiologie et de santé publique 2001; 49: 135-145.

20. Dafni UG, et al. Algorithm for statistical detection of peaks - syndromic surveillance system for the Athens 2004 Olympic Games. Morbidity and Mortality Weekly Report 2004; 53 (Suppl.): 86-94.

21. Epidemiological Consultation Team. Surveillance system in place for the 2006 Winter Olympic Games, Torino, Italy, 2006. Eurosurveillance Weekly 2006; 11(2): E060209.

22. Gundlapalli AV, et al. Hospital electronic medical record-based public health surveillance system deployed during the 2002 Winter Olympic Games. American Journal of Infection Control 2007; 35: 163-171.

23. Osaka K, Takahashi H, Ohyama T. Testing a symptombased surveillance system at high-profile gatherings as a preparatory measure for bioterrorism. Epidemiology and Infection 2002; 129: 429-434.

24. Meyer N, et al. A multi-data source surveillance system to detect a bioterrorism attack during the G8 Summit in Scotland. Epidemiology and Infection 2008; 136: 876885. 